\title{
On the Error Rate and Delay Performance Analysis of OFDM based Cooperative Protocol for 802.11 Networks
}

\author{
Irfan Ahmed \\ College of Computers and Information Technology \\ Taif University, Taif, Kingdom of Saudi Arabia \\ i.ahmed@tu.edu.sa
}

\begin{abstract}
We develop and analyze a distributed space-frequency block code-orthogonal frequency division multiplexing protocol for cooperative communications in 802.11 networks. Space frequency block codes (SFBC) are spread over OFDM subcarriers instead of OFDM symbols to compensate for the small coherence time. Medium access control (MAC) layer packet retransmission limit has been used as an actuator for transmit cooperative diversity initialization. Transmit diversity is provided by the relays in close proximity to source node. Closed form expressions are obtained for packet error rate (PER) and average delay for the proposed scheme in Nakagami-m fading channels. This cooperative scheme achieves lower signal-to-noise ratio (SNR) values for desired packet error rate and markedly improves the average delay per packet compared to the direct transmissions at low SNR regime. Finally, the results of computer simulations are included to demonstrate the efficacy of the proposed scheme and to verify the accuracy of analytical expressions.
\end{abstract}

\section{Indexing terms/Keywords}

Automatic repeat request (ARQ), cooperative transmit diversity, OFDM, wireless networks.

\section{Academic Discipline And Sub-Disciplines}

Telecommunication engineering; Wireless communications; Wireless LAN;

\section{SUBJECT CLASSIFICATION}

Digital Communications

\section{TYPE (METHODIAPPROACH)}

Simulations and analysis

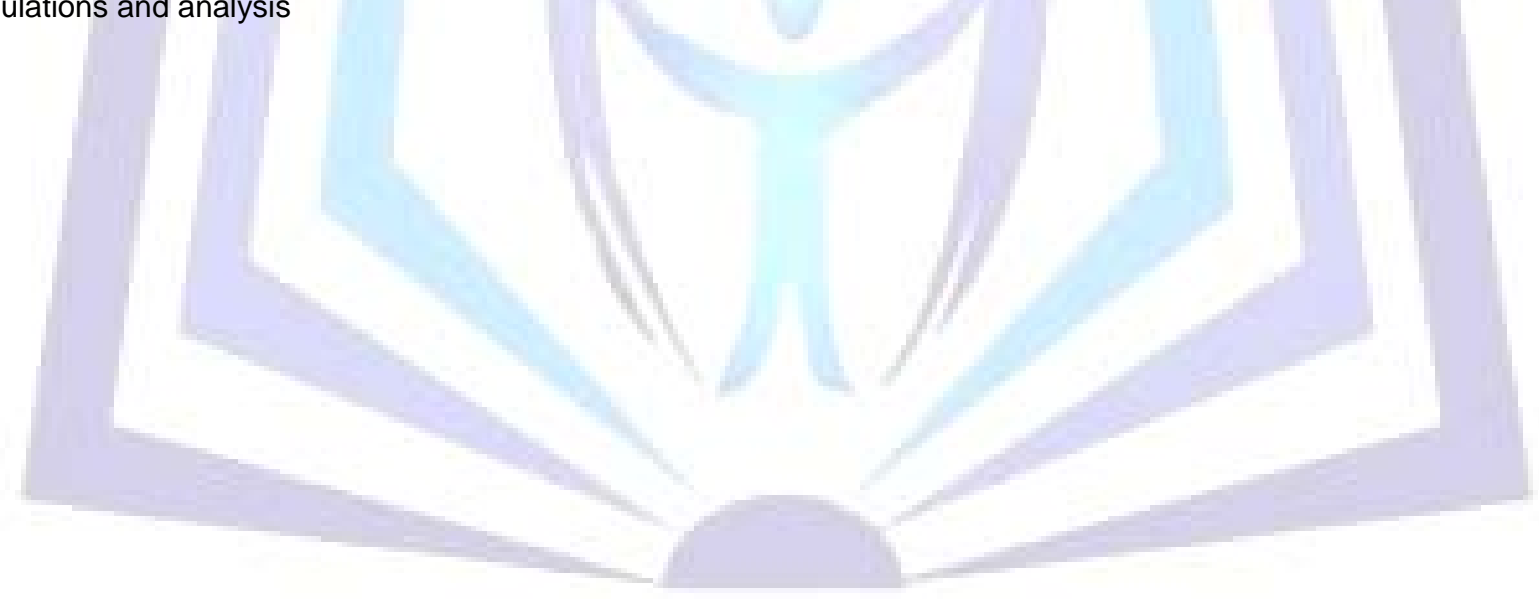

\section{Council for Innovative Research}

Peer Review Research Publishing System

Journal: INTERNATIONAL JOURNAL OF COMPUTERS \& TECHNOLOGY

Vol 10, No 2 


\section{INTRODUCTION}

Recent researches have shown that the spatial diversity can be leveraged at MAC and PHY layers to provide energy efficient transmission. Multiple input multiple output (MIMO) realized this spatial diversity. MIMO systems require multi antenna devices, which may not be feasible in some devices due to their small physical size, e.g., sensor nodes. This area of thrust then brought a new arena of cooperative diversity where the diversity gain is achieved via virtual antenna array in a distributed manner (cluster of relay nodes) called cooperative or relay communications.

In general, two types of relays are possible: decoding relays, which must successfully decode the message before forwarding (decode-and-forward), and amplifying relays, which simply repeat an amplified version of the received signal without first decoding (amplify-and-forward) [1]. Hybrid relays may adaptively switch between decoding and amplifying modes [2] opportunistically. Laneman et al. [1] indicated that adaptive decode-and-forward strategies offer the same performance as fixed amplify-and-forward. Meng Yu et al. [3] have shown that the decode-and-forward scheme is slightly better than the amplify-and-forward in error probability and mutual information analysis. More importantly, in order to fully realize the diversity order promised by the theory, a source node should probably partner with one that is as close as possible [3].

We consider the case where the relay terminals decode the received symbols and retransmit them. This strategy is known as Decode and Forward (DF). Talking about DF DSFBC, it is important to clarify first the main differences between DSFBC and SFBC, besides the obvious property that the multiple transmit antennas in the DSFBC scheme belong to different radio nodes. The main differences are the following: i) in the DSFBC there are errors in the link between the transmit antennas (source $S$ and relay $R$ nodes), whereas of course there are no errors at the transmitter in SFBC; ii) even if $S$ and $R$ transmit synchronously, the signals arriving at destination node $D$ might be asynchronous, because of the different delays from $S$ and $R$. To limit this second source of error, we assume that a radio node is chosen as a relay only if it is sufficiently close to the source, so that the relative delay can be incorporated within the cyclic prefix (CP) appended to each transmitted block without any major efficiency loss.

Most of the cooperative techniques presented in the literature can be classified into two categories, namely, physicallayer cooperation and MAC-layer cooperation. Physical-layer cooperation techniques, or cooperative relaying, involves nodes in the vicinity of a single wireless link that relay overheard packets to the link's receiver (or, with coded cooperation transmit error-correcting redundant information that allows the receiver to recover the packet by jointly decoding the relayed signals) [4]-[10]. On the basis of initialization, the existing cooperative MAC protocols can be classified into proactive schemes [11-14] and reactive schemes $[15,16]$. In the former, the cooperation of the partner(s) is always provided by either the prearranged optimal $[14,17]$ or the random $[11-13]$ helper(s) before the acknowledgment (ACK) from the receiver; while in the latter, the help from the partner(s) is initiated only when the negative acknowledgment (NACK) is received/detected. In [11, 12], two similar protocols (called Coop-MAC and rDCF) based on the IEEE 802.11 distributed coordinated function (DCF) are proposed to mitigate the throughput bottleneck caused by low-data-rate nodes. A high-rate node is allowed to help a low-rate node through two-hop transmission. Through joint routing and cooperation a cross-layer approach is introduced in [13]. Clusters of nodes near each transmitter form virtual multiple-input singleoutput (VMISO) link to a receiver on the routing table and as far as possible to the transmitter. Space-time codes are utilized to support transmission over a long distance, thus reducing the number of transmission hops and improving communication reliability. $\mathrm{H}$. Shan et al. [14] propose a busy-tone based cross-layer cooperative MAC (CTBTMA) protocol Adaptive modulation and coding (AMC) and multimode transmission are scheduled together according to the channel condition to improve the network throughput. The use of busy tones helps to solve collisions in a cooperation scenario and to address the optimal helper selection problem. Reactive schemes $[15,16]$ have a similar strategy, which let neighbor(s) (overhearing the packet) retransmit the packet instead of the source node when the NACK is detected.

From the viewpoint of space-frequency cooperation, the longer symbol duration along with the use of cyclic prefix makes the system robust to timing errors. However, at the same time, longer symbol duration causes OFDM signals to become more sensitive to frequency offsets. Timing synchronization and frequency offset estimation and correction for cooperative OFDM system has been described briefly in [18] and are not the focus of this paper.

In this paper, we design a space-frequency cooperative system, based on, PHY layer OFDM, distributed SFBC, and MAC layer RTS/CTS/ACK (Request-to-send/Clear-to-send/Acknowledgement) protocol and evaluate the performance of the proposed scheme. This type of node communication results in some additional delay and overhead compared to the conventional ARQ or previous cooperative diversity protocols. However, considering that the transmission is performed in the unit of packet (over one hundred symbols per packet; for example), this overhead can be neglected since it only requires several bits.

The remaining of the paper is organized as follows. Section II describes the signal, system and the channel model for wireless network under consideration. Section III contains Truncated ARQ based DSFBC-CO-OFDM scheme. Next, the 
performance analysis of the proposed cooperative communications scheme is presented in Section IV. Section $V$ shows the MATLAB ${ }^{\circledR}$ simulation results, followed by conclusions in Section VI.

\section{SIGNAL SYSTEM AND CHANNEL MODEL}

We consider the system model shown in Fig. 1 where source node $S$ along with $N_{r}$ relay nodes form $\left(N_{r}+1\right) \times 1$ cooperative communication link with the destination node $D$. We model this $N_{r}$ by a random variable with range $\left[0, \Upsilon_{r}^{\max }\right]$, where $\Upsilon_{r}^{\max }$ is the maximum retransmission limit. Further, it is assumed that receiver has perfect channel state information through the use of training sequence at PHY PMD (physical medium dependent).

The source node sends a packet $x_{t+1}^{s}, \ldots, x_{t+\xi}^{s}$ with transmission power $P^{s}$ per OFDM symbol, where $\xi=L_{p} / b k_{\wp_{d}}$ is the number of OFDM symbols per packet with a total packet length of $L_{p}$ bits and a modulation level of $b=\log _{2} M$ bits. Each OFDM symbol carries $k \wp_{d} M$-QAM data symbols ( $k$ is the number of subcarriers per OFDM symbol). The packet received by the destination node during direct transmission is given by:

$$
y_{\text {packet }}^{\text {sd }}=\left[y_{t+1}^{s d}, \ldots, y_{t+\xi}^{s d}\right]
$$

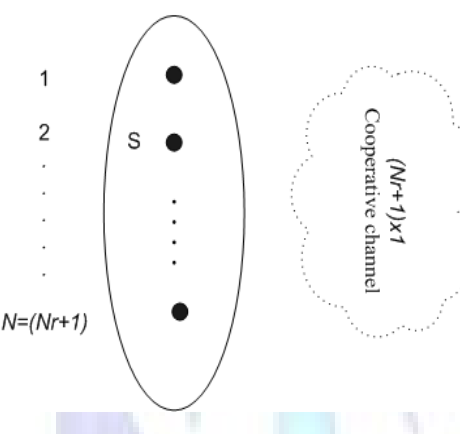

Figure 1. System model

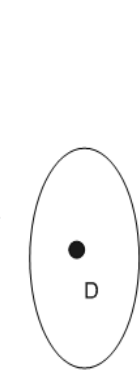

where $y_{t+i}^{s d}=h_{t+i}^{s d} x_{t+i}^{s}+n_{0}, i=1, \ldots, \xi$ and the channel gain $h_{t+i}^{s d}$ is assumed to be a Nakagami- $m$ random variable with mean ${ }_{t+i}^{s d}$ and variance $\sigma_{0, t+i}^{s d} . n_{0}$ represents additive white Gaussian noise with zero mean and variance $\aleph_{0}$. Packet received by $n^{\text {th }}$ relay node, $n=1, \ldots, N$, is given by

$$
y_{\text {packet }}^{s r_{n}}=\left[y_{t+1}^{s r_{n}}, \ldots, y_{t+\xi}^{s r_{n}}\right]
$$

where $y_{t+i}^{s r_{n}}=h_{t+i}^{s r_{n}} X_{t+i}^{s}+n_{0}^{s r_{n}}, i=1, \ldots, \xi$.

The received packet at the destination node is given by

$$
\begin{aligned}
& y_{\text {packet }}^{d}=\left[y_{t+1}^{d}, \ldots, y_{t+\xi^{\prime}}^{d}\right] \\
& y_{t+i}^{d}=h_{0}^{s d} \chi_{t+i}^{s}+n_{0}^{s d}+\sum_{n=1}^{N} h_{n}^{r d} \chi_{t+i}^{r d, n}+n_{0}^{r d, n}
\end{aligned}
$$

Where $\xi^{\prime}=\xi k \wp_{d} \Upsilon_{r} / R_{c}$ with $\Upsilon_{r}$ number of retransmissions and $R_{c}$ SFBC code rate. $\chi_{t+i}^{s}$ and $\chi_{t+i}^{r d, n}$ are the spacefrequency block coded symbols transmitted by source node and $n^{\text {th }}$ relay node, respectively. The fading coefficients $h_{n}^{r d}$ are modeled by Gamma random variable and $n_{0}^{r d, n}$ is the additive White Gaussian noise with zero mean and variance $\sigma_{0}^{r d, n}$.

In general, at time $t$, for $N_{r}$ cooperative nodes the $\left(N_{r}+1\right) \times L$ space-frequency code word matrix [11] is given by 


$$
X_{t}=\left[\begin{array}{cccc}
x_{t, 1}^{1} & x_{t, 2}^{1} & \cdots & x_{t, L}^{1} \\
x_{t, 1}^{2} & x_{t, 2}^{2} & \cdots & x_{t, L}^{2} \\
\vdots & \vdots & \ddots & \vdots \\
x_{t, 1}^{N_{r}+1} & x_{t, 2}^{N_{r}+1} & \cdots & x_{t, L}^{N_{r}+1}
\end{array}\right]
$$

where the $i$-th row $x_{t}^{i}=x_{t, 1}^{i}, x_{t, 2}^{i}, \ldots, x_{t, L}^{i}, i=1,2, \ldots, N_{r}+1$, is the data sequence for the $i$-th relay. We assume that the subcarrier length $K$ is an integer multiple of SFBC code word length $L$. Signals $x_{t}^{i}=x_{t, 1}^{i}, x_{t, 2}^{i}, \ldots, x_{t, L}^{i}, i=1,2, \ldots, N_{r}+1$ are OFDM modulated on $L$ different OFDM subcarriers and transmitted from the $i$-th relay simultaneously during one OFDM symbol, where $x_{t, k}^{i}$ is sent on the $k$-th OFDM subcarrier.

Assume that $n_{s}$ symbols are transmitted by the transmission matrix. Then the code rate $R_{c}$ is defined as $R_{c}=n_{s} / L$, where $L$ is the number of subcarriers for transmission of one block of coded symbols.

The wireless communication is assumed to be over Nakagami- $m$ fading channel. Let $\gamma$ denote the instantaneous SNR defined by $\gamma \triangleq \alpha^{2}\left(E_{s} / N_{0}\right) / N_{r} R_{c}$ where $\alpha$ is the fading amplitude, $E_{s}$ is the energy per symbol, and $N_{0}$ is the one-sided noise spectral density. Probability density function (pdf) of $\alpha$ is given by

$$
p_{\alpha}(\alpha)=\frac{2}{\Gamma(m)}\left(\frac{m}{\Omega}\right)^{m} \alpha^{2 m-1} e^{-m \alpha^{2} / \Omega}, \alpha \geq 0
$$

Where $\Gamma(\cdot)$ is the gamma function, $\Omega \triangleq E\left[\alpha^{2}\right]$ defined as mean square value, and $m$ is the fading severity parameter from $1 / 2$ to $\infty$. Then the pdf and moment generating function (MGF) of instantaneous SNR $\gamma$ for $n$ relays assisted are given by, respectively

$$
\begin{gathered}
p_{\gamma}(\gamma)=\frac{1}{\Gamma(m)}\left(\frac{m}{\bar{\gamma}}\right)^{m} \gamma^{m-1} e^{-m \gamma / \bar{\gamma}}, \gamma \geq 0 \\
\phi_{\gamma}(s) \triangleq \int_{0}^{\infty} e^{-s \gamma} p_{\gamma}(\gamma) d \gamma=\left(1+\frac{s \bar{\gamma}}{m}\right)^{-m}, m \geq \frac{1}{2}
\end{gathered}
$$

Where $\bar{\gamma} \triangleq \Omega E_{s} / N_{0} R_{c}(n+1)$ denotes the average SNR per symbol.

\section{Distributed Space-frequency Signal}

In the Cooperative-OFDM system, MAC layer transmission of each frame involves two phases. Phase I is associated with normal transmission of data frame from source to the relay and destination without space-frequency coding. If the destination succeeds in decoding the Phase I data frame with the broadcasting of ACK frame, the following Phase II (SFBC cooperation) is ignored at the source and the relay. Otherwise, after the frame check sequence (FCS) error detection at destination followed by ACK timeout, the source and relays start SFBC transmission.

For simplicity, we consider one relay and the Alamouti code to construct space-frequency-coded signals in cooperation phase. Each subcarrier pair in OFDM symbol form a space-frequency coded block, this can be easily extended to more than one cooperative relays using Tarokh STBC/SFBC codes. Let $X_{S}[k], X_{S}[k+1]$ be the space-frequency encoded symbol

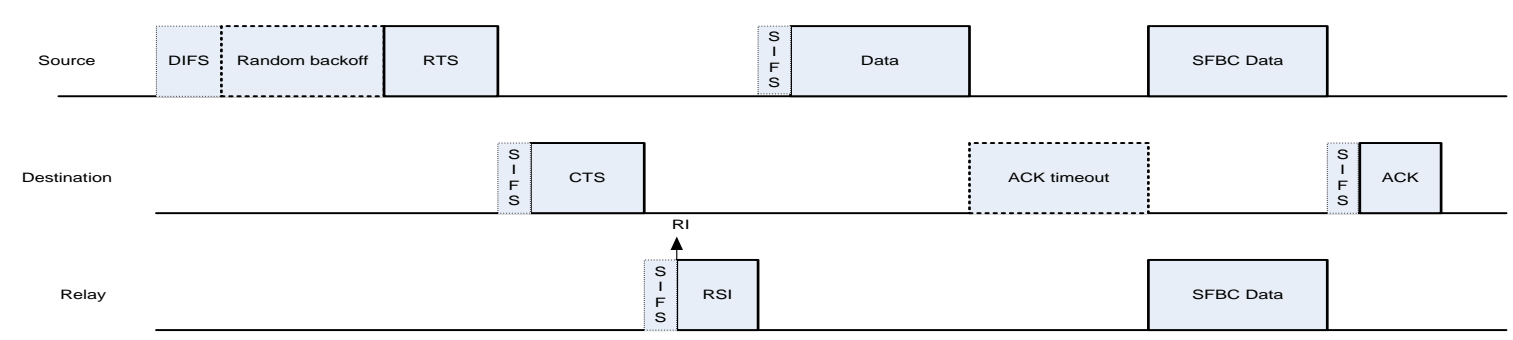


Frame sequence for 1 cooperative relay

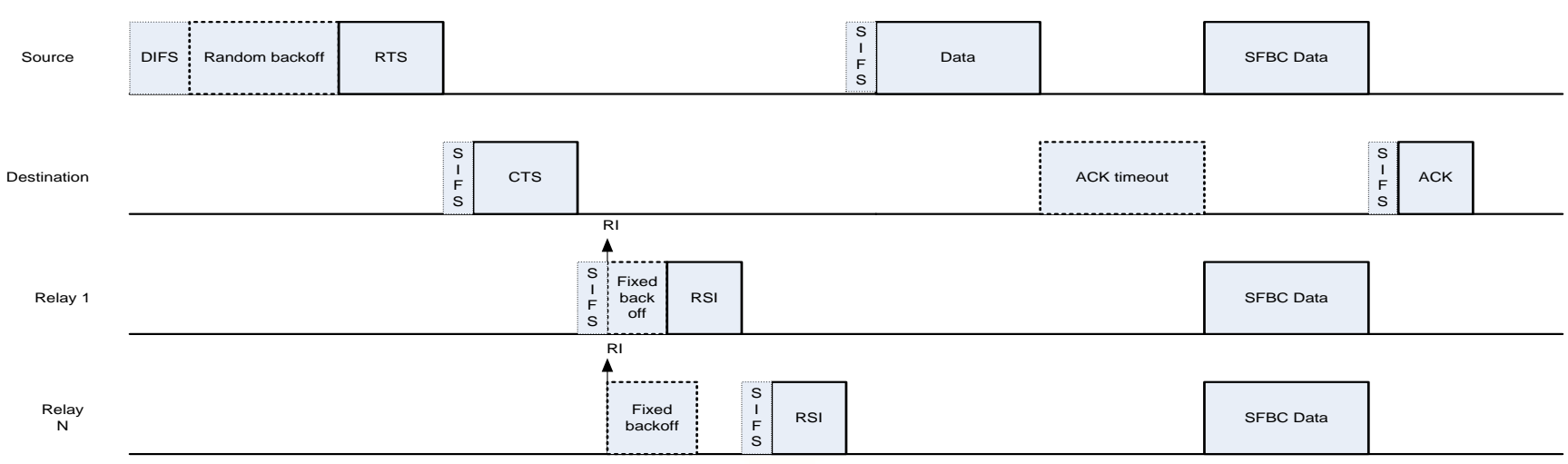

Figure 2. Frame sequence for $\mathrm{N}$ cooperative relays

transmitted from source $S$ in $k$-th and $(k+1)$-th subcarrier for $m$ th OFDM symbol. Similarly, $X_{R}[k], X_{R}[k+1]$ be the spacefrequency encoded symbol transmitted from relay $R$. The space-frequency block code in subcarrier pair can be expressed in matrix form as follows

$$
\left[\begin{array}{ll}
X_{S}[k] & X_{S}[k+1] \\
X_{R}[k] & X_{R}[k+1]
\end{array}\right]=\left[\begin{array}{cc}
S_{1, S}[k] & -S_{2, S}^{*}[k+1] \\
S_{2, R}[k] & S_{1, R}^{*}[k+1]
\end{array}\right]
$$

The received symbols $R[k]$ and $R[k+1]$ at the destination can be written as

$$
\left[\begin{array}{c}
R[k] \\
R^{*}[k+1]
\end{array}\right]=\left[\begin{array}{cc}
\mathrm{H}^{S D}[k] & \mathrm{H}^{R D}[k] \\
\mathrm{H}^{* R D}[k+1] & -\mathrm{H}^{* S D}[k+1]
\end{array}\right]\left[\begin{array}{c}
S_{1, S}[k] \\
S_{2, R}[k+1]
\end{array}\right]+\left[\begin{array}{c}
\mathbb{N}[k] \\
\mathbb{N}^{*}[k+1]
\end{array}\right]
$$

Where $\mathrm{H}^{l}[k]$ denotes the channel impulse response of the link / for the subcarrier $k$ during $m$-th OFDM symbol, and $\mathbb{N}[k]$ is the additive White Gaussian noise (AWGN) with zero mean and variance of $\sigma^{2}$. Assuming the number of subcarriers is equal to the codeword length $L$ and flat fading over the $L$ subcarriers. An alternative technique is to use spatial diversity coding on a subcarrier basis across OFDM symbol in time [1]. However, this requires that the channel remain constant over $L$ OFDM symbol periods. Since the duration of an OFDM symbol $\left(\left(N \_\right.\right.$data $\left.\left.+N \_p i l o t+N \_c p\right) / B W\right)$ is usually large, this may be impractical.

For the decoding of space-frequency-coded symbols, the destination assumes $\mathrm{H}^{l}[k]=\mathrm{H}^{l}[k+1]$. If channel estimates $\hat{\mathrm{H}}^{l}[k], l=S D, R D$ are available, then destination can obtain soft decision on $S_{1, S}[k]$ and $S_{2, S}[k]$ using the maximum likelihood rule as [22]

$$
\left[\begin{array}{c}
\widehat{S}_{1, S}[k] \\
\widehat{S}_{2, S}[k]
\end{array}\right]=\frac{1}{\widehat{\Theta}[k]}\left[\begin{array}{cc}
\hat{\mathrm{H}}^{* S D}[k] & \hat{\mathrm{H}}^{R D}[k] \\
\hat{\mathrm{H}}^{* R D}[k+1] & -\hat{\mathrm{H}}^{S D}[k+1]
\end{array}\right] \times\left[\begin{array}{c}
R[k] \\
R^{*}[k+1]
\end{array}\right]
$$

Where $\widehat{\Theta}[k] \triangleq\left|\hat{H}^{S D}[k]\right|^{2}+\left|\widehat{H}^{R D}[k]\right|^{2}$

These received symbols are converted into bits through M-QAM demodulator followed by the PHY PMD packet formation.

\section{TRUNCATED ARQ BASED DSFBC-CO-OFDM SCHEME}

In this section we propose a cross layer cooperative MAC scheme which takes into account the MAC layer ARQ, PHY layer DSFBC and cooperative OFDM. The proposed scheme is based on IEEE 802.11 DCF with cooperative communications realization. Fig. 2, Fig. 3 and Algorithm I explain the proposed IEEE 802.11 DCF based cooperative MAC protocol.

Relay nodes are selected on following condition 


$$
\begin{aligned}
& \frac{1}{R_{s d}} \geq\left(\frac{1}{R_{n}}+N \times S I F S+N \times R S I\right), \\
& \text { where } R_{n}=\min \left(R_{s r, n}, R_{r d, n}\right)
\end{aligned}
$$

and $R_{s d}, R_{s r}, R_{r d}$ are transmission rates of source-destination, source-relay, and relay-destination, respectively. SIFS is short inter-frame spacing and is defined in 802.11.

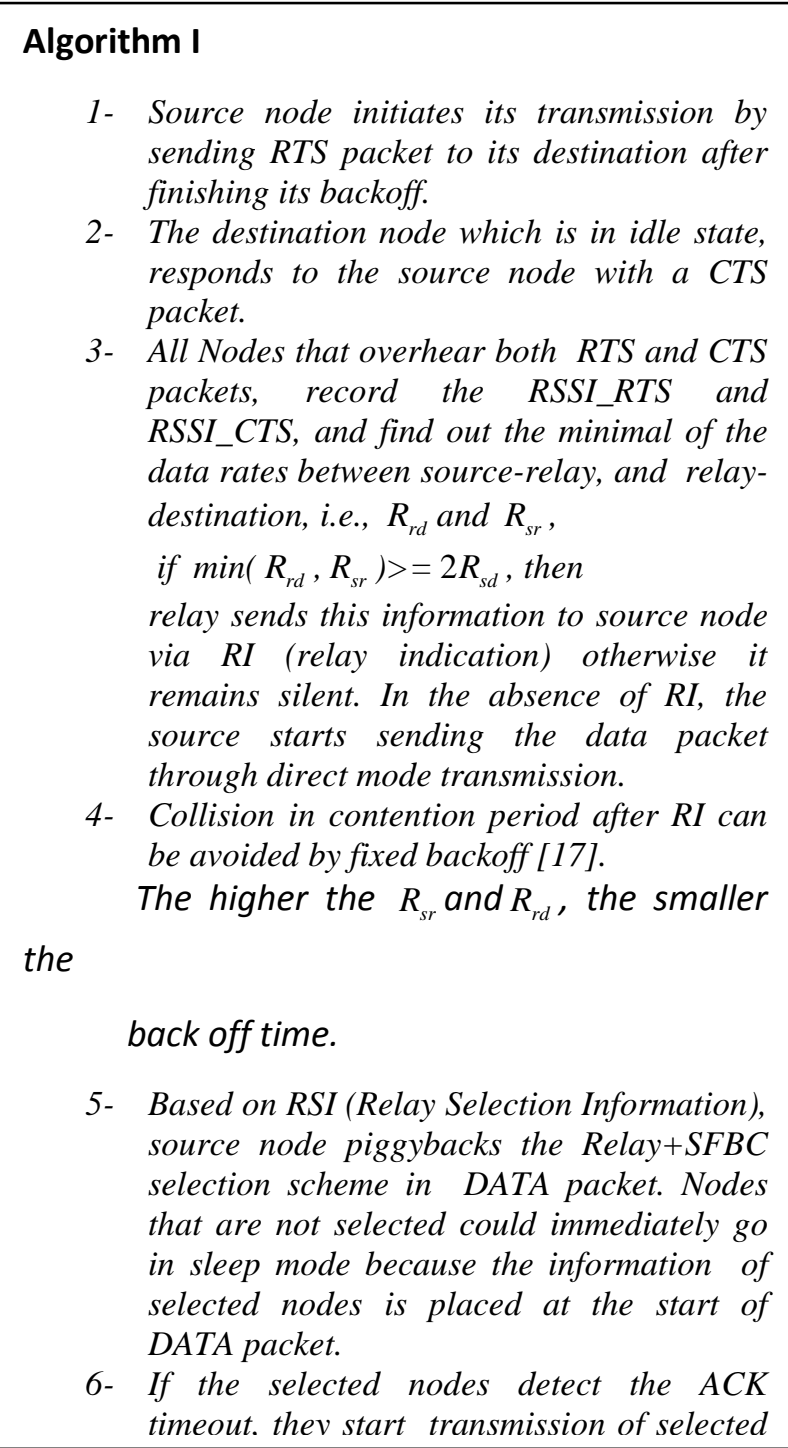

\section{PERFORMANCE ANALYSIS}

With the assumption that the length of PHY PLCP (physical layer convergence protocol) packet is $L_{p}$ and a square $M$ QAM is adopted with $b=\log _{2} M$ bits/symbol, we obtain the relations for error rates and average delays as follows:

\section{Error Rates}

The average SER for $n$-relay assisted M-QAM signal is given by [19]

$$
P_{s}(e)=\frac{4 q}{\pi} \int_{0}^{\pi / 2} \phi_{\gamma}^{-(n+1)}\left(\frac{p}{\sin ^{2} \theta}\right) d \theta-\frac{4 q^{2}}{\pi} \int_{0}^{\pi / 4} \phi_{\gamma}^{-(n+1)}\left(\frac{p}{\sin ^{2} \theta}\right) d \theta
$$

Where $p=3 /(2(M-1))$, and $q=1-1 / \sqrt{M}$,

Evaluation of the above integral results in the following: 


$$
\begin{aligned}
P_{S}(e) & =\frac{2 q \Gamma\left(m(n+1)+\frac{1}{2}\right.}{\sqrt{\pi} \Gamma(m(n+1)+1)} \phi_{\gamma}(p) \cdot{ }_{2} F_{1}\left(m(n+1), \frac{1}{2} ; m(n+1)+1 ; \frac{1}{1+\frac{p \bar{\gamma}}{m(n+1)}}\right) \\
& -\frac{2 q^{2}}{\pi(2 m(n+1)+1)} \phi_{\gamma}(2 p) \cdot F_{1}\left(1, m(n+1), 1 ; m(n+1)+\frac{3}{2} ; \frac{1+\frac{p \bar{\gamma}}{1+\frac{2 p(n+1)}{m(n+1)}}, \frac{1}{2}}{2}\right)
\end{aligned}
$$

Where ${ }_{2} F_{1}(., . ; . ;$.$) and F_{1}(., ., . ; . ; . .$.$) are the Gauss Hypergeometric function [20, eq.2.12(1)] and Appell Hypergeometric$ function [20, eq.5.8(5)], respectively.

The mathematical software used is Matlab, which contains the Gauss Hypergeometric function but it does not include the Appell Hypergeometric function. This function has been implemented by using their series representation [21, eq.(9.180.1)]

$$
F_{1}\left(a, b, b^{\prime} ; c ; x, y\right)=\sum_{n=1}^{\infty} \sum_{k=1}^{\infty} \frac{(a)_{n+k}(b)_{n}\left(b^{\prime}\right)_{k}}{(c)_{n+k} n ! k !} x^{n} y^{k}
$$

where

$(a)_{n} \triangleq \Gamma(a+n) / \Gamma(a)$ is the Pochhammer symbol [21].

The probability of packet error at the destination for ARQ initialized cooperative communication, can be obtained by combining the law of conditional probability and law of total probability as

$$
\begin{aligned}
\mathrm{P}_{p}^{A R Q}(e) & =\operatorname{Pr}\left(e \mid\left(1 \leq \Upsilon_{r} \leq \Upsilon_{r}^{\max }\right)\right) \operatorname{Pr}\left(1 \leq \Upsilon_{r} \leq \Upsilon_{r}^{\max }\right) \\
& +\operatorname{Pr}\left(e \mid\left(1 \leq \Upsilon_{r} \leq \Upsilon_{r}^{\max }\right)^{\prime}\right) \operatorname{Pr}\left(1 \leq \Upsilon_{r} \leq \Upsilon_{r}^{\max }\right)^{\prime}
\end{aligned}
$$

$\operatorname{Pr}\left(e \mid\left(1 \leq \Upsilon_{r} \leq \Upsilon_{r}^{\max }\right)\right)=1$, given that CRC error has been detected.

$\operatorname{Pr}\left(e \mid\left(1 \leq \Upsilon_{r} \leq \Upsilon_{r}^{\max }\right)^{\prime}\right)=0$, given that no CRC error has been detected.

Retransmission tries $\Upsilon_{r}$ can vary in the range [0- $\Upsilon_{r}^{\max }$ ]

$$
\mathrm{P}_{p}^{A R Q}(e)= \begin{cases}{\left[1-\left(1-P_{s}(E)\right)^{L_{p} / b}\right]^{\Upsilon_{r}},} & , 1 \leq \Upsilon_{r} \leq \Upsilon_{r}^{\max } \\ 0 & , \Upsilon_{r}=0\end{cases}
$$

Packet loss is occurred when retransmission exceeds the maximum limit $\Upsilon_{r}^{\max }$, with packet loss probability given by:

$$
\mathrm{P}_{p}^{A R Q}(\text { loss })=\left[1-\left(1-P_{s}(E)\right)^{L_{p} / b}\right]^{\Upsilon_{r}^{\max }+1}
$$

\section{Average Delay}

The average delay at MAC layer for $\bar{\Upsilon}_{r}$ average number of retransmissions can be computed as

$$
\mathfrak{D}_{A v g}=\frac{\bar{\Upsilon}_{r}^{2} L_{p}^{2}}{b\left(L_{p}-M_{O H}\right)} \frac{1}{P_{p}(s)}
$$

Where $M_{O H}$ is MAC overhead and $P_{p}(s)$ is the MAC level packet success rate, respectively.

A packet error occurs if at least one of the $\kappa$ bits is erroneous. Since the nodes are spatially separated, no correlation has been observed and the bits in a packet are independent from each other, which yields the following PER 


$$
P_{p}(e)=1-\left(1-P_{b}(e)\right)^{L_{p}}
$$

Assuming bits are Gray mapped onto the symbol, i.e., adjacent symbol constellation points differ by one bit [22], then $P_{b}(e)=P_{s}(e) / \log _{2}(M)$.

MAC level packet success rate is given by

$$
P_{p}(s)=1-P_{p}^{\text {direct }}(e)\left(P_{p}^{\text {retrans }}(e)\right)^{\Upsilon_{r}^{\max }}
$$

and the average number of retransmission per packet is given as

$$
\begin{aligned}
\bar{\Upsilon}_{r}= & 1-P_{p}^{\text {direct }}+\sum_{i=2}^{\Upsilon_{r}^{\max }} i \cdot\left(P_{p}^{\text {retrans }}\right)^{i-2}\left(1-P_{p}^{\text {retrans }}\right) P_{p}^{\text {direct }} / R_{c} \\
& +\left(\Upsilon_{r}^{\max }+1\right) \cdot\left(P_{p}^{\text {retrans }}\right)^{\Upsilon_{r}^{\max }-1} \cdot P_{p}^{\text {direct }} / R_{c}
\end{aligned}
$$

\section{SIMULATION RESULTS}

In this section, we provide computer simulations using MATLAB ${ }^{\circledR}$. In this simulations we have used packet length $L_{p}=100$, QPSK modulation, Nakagami parameter $\mathrm{m}=1$, and maximum retransmission tries $\Upsilon_{r}^{\max }=4$ (similar to 802.11).

ARQ based average SER is shown in Fig. 4 with different number of retransmission tries. First relay is selected when destination detects FCS error. If destination is not succeeded in FCS decoded again, second relay is selected to cooperate with source and first relay, and so on. It can be seen from the figure that there is an obvious diversity gain with increasing number of relays but at the same time, there is a reduction in throughput due to the delay incurred in each retransmission. Destination node can receive the transmitted frame even in harsh fading conditions because of the given diversity gain. Fig. 5 shows the MAC layer packet error rate. At a given received SNR, the average PER decreases with the increasing number of relays/retransmissions. Packet loss probability of proposed scheme and direct transmission scheme is shown in Fig. 6. Space-frequency based coded scheme provides about $6 \mathrm{~dB}$ gain over direct transmission with one relay at packet loss probability of $10^{-2}$. Using packet loss probability and acceptable time delay, one can restrict the number of retransmissions. Average delays for proposed scheme is shown in Fig. 7. For small values of received SNR, direct transmission results in more delay than retransmission based cooperative scheme, it's due to the large number of retransmissions from source only. Our proposed scheme gives a dominant performance gain over direct transmission in low SNR regime.

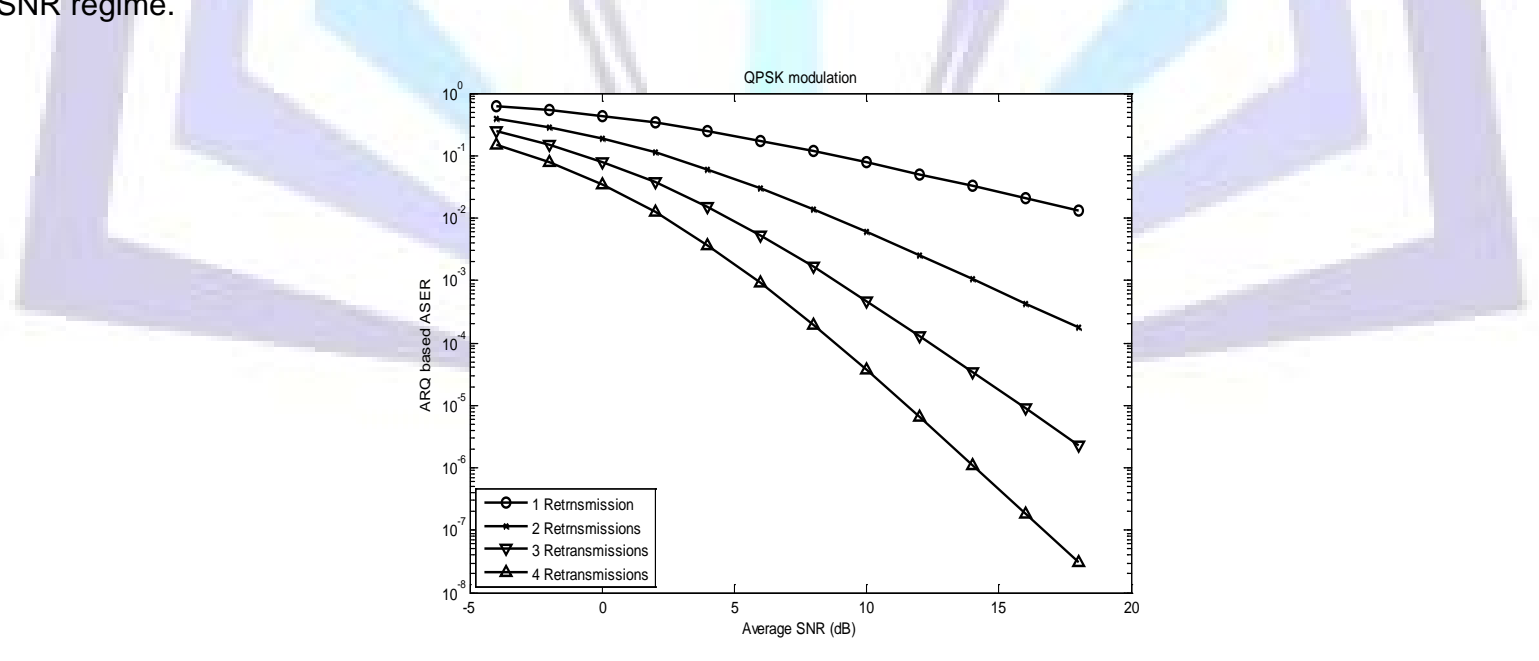

Figure 3. ASER Vs SNR with QPSK modulation 


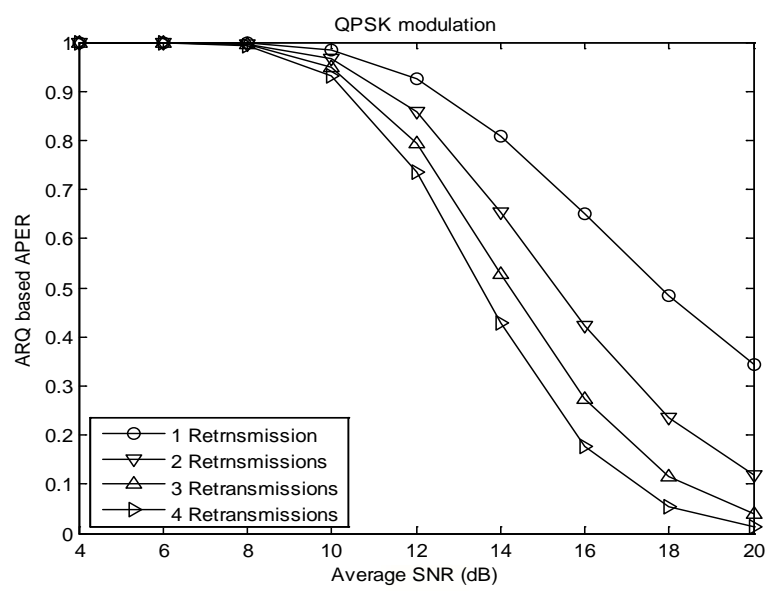

Figure 4. ARQ based PER Vs SNR for QPSK modulation

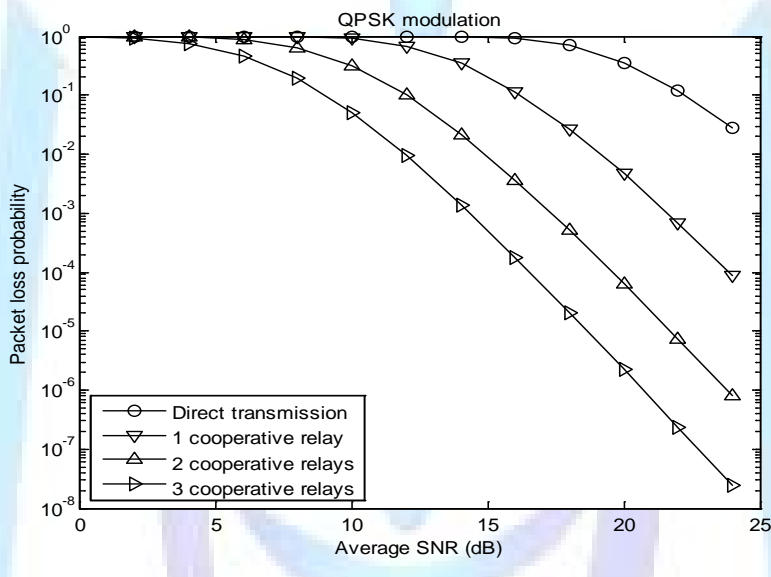

Figure 5. Packet loss probability Vs SNR

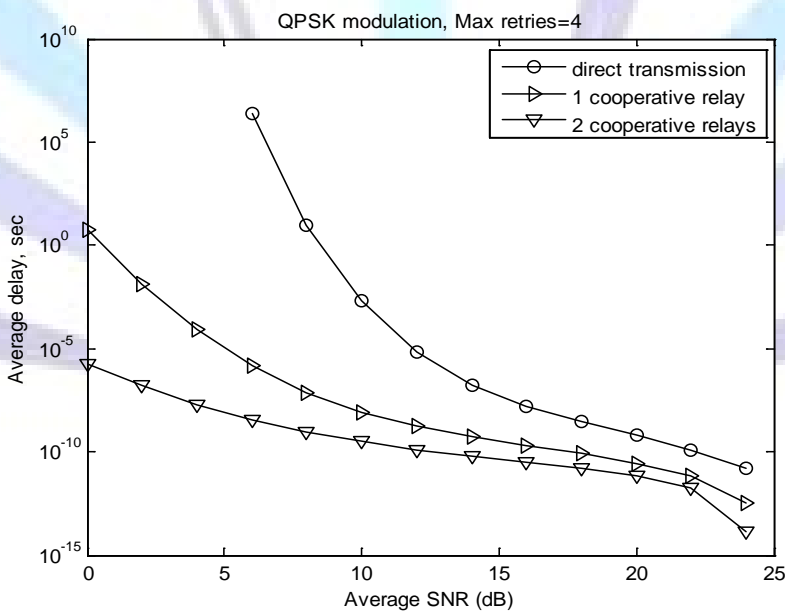

Figure 6. Average delay variations with received SNR

\section{CONCLUSIONS}

In this paper, we have presented the symbol and packet error rate performance analysis of ARQ initialized DSFBCOFDM transmit diversity cooperative communications system in Nakagami- $m$ fading channel. Relays are opportunistically selected through MAC layer ARQ protocol which eliminates the feedback overhead of PHY layer relay selection. Simulation results show that SER and PER performance are improved with proposed cooperative scheme with much smaller delay per packet as compared to direct transmission. This work can be extended to spatial multiplexing and the switching between diversity and spatial multiplexing depending upon the channel condition through Demmel number. 


\section{REFERENCES}

[1] J. N. Laneman, D. N. C. Tse, and G. W.Wornell, "Cooperative diversity in wireless networks: efficient protocols and outage behavior," IEEE Trans. Inform. Theory. vol. 50, pp.3062-3080, Dec. 2004.

[2] B. Zhao and M. C. Valenti, "Some new adaptive protocols for the wireless relay channel," in Proc. Allerton Conf Commun., Control, Comput.,Allerton, IL, Oct. 2003.

[3] Meng Yu; Jing Li; "Is amplify-and-forward practically better than decode-and-forward or vice versa?" Acoustics, Speech, and Signa Processing, 2005. Proceedings. (ICASSP '05). IEEE International Conference on Volume 3, 1823 March 2005 Page(s):iii/365 - iii/368

[4] A.S. Ibrahim, A.K. Sadek, W. Su, and K.J.R. Liu. Cooperative communications with relay selection: when to cooperate and whom to cooperate with? IEEE Transactions on Wireless Communications, 7(7):2814-2827, July 2008.

[5] L. Sun, T. Zhang, L. Lu, and H. Niu. Cooperative communications with relay selection in wireless sensor networks. IEEE Trans. on Consumer Electronics, 55(2):513-517, May 2009.

[6] J.L. Vicario, A. Bel, J.A. Lopez-Salcedo, and G. Seco. Opportunistic relay selection with outdated CSI: Outage probability and diversity analysis. IEEE Transactions on Wireless Communications, 8(6):2872-2876, June 2009.

[7] F. Xu, F.C.M. Lau, Q.F. Zhou, and D-W. Yue. Outage performance of cooperative communication systems using opportunistic relaying and selection combining receiver. IEEE Signal Processing Letters, 16(2):113-116, April 2009.

[8] A. Sendonaris, E. Erkip, and B. Aazhang. User cooperation diversity (parts I,II). IEEE Transactions on Communications, 51(11):1927-1948, November 2003.

[9] T.E. Hunter and A. Nosratinia. Diversity through coded cooperation. IEEE Transactions on Wireless Communications 5(2):283-289, February 2006.

[10] P. Elia, K. Vinodh, M. Anand, and P.V. Kumar. D-MG tradeoff and optimal codes for a class of AF and DF cooperative communication protocols. IEEE Transactions on Information Theory, 55(7):3161-3185, July 2009.

[11] P. Liu et al., "CoopMAC: A Cooperative MAC for Wireless LANs," IEEE JSAC, vol. 25, no. 2, Feb. 2007, pp.340-54.

[12] H, Zhu and G. Cao, "rDCF: A Relay-Enabled Medium Access Control Protocol for Wireless Ad Hoc Networks," IEEE Trans. Mobile Comp., vol. 5, no. 9, Sept. 2006, pp. 1201-14.

[13] G. Jakllari et al., "A Framework for Distributed Spatio-Temporal Communications in Mobile Ad Hoc Networks," Proc. IEEE INFOCOM, 2006.

[14] H. Shan et al., "Cross-Layer Cooperative Triple Busy Tone Multiple Access for Wireless Networks," Proc. IEEE GLOBECOM, 2008.

[15] B. Zhao and M. C. Valenti, "Practical Relay Networks: A Generalization of Hybrid-ARQ," IEEE JSAC, vol. 23, no.1, Jan. 2005, pp. 7-18.

[16] M. Dianati et al., "A Node Cooperative ARQ Scheme for Wireless Ad Hoc Networks," IEEE Trans. Vehic. Tech., vol. 55, no. 3, May 2006, pp. 1032-44.

[17] A. Bletsas et al., "A Simple Cooperative Diversity Method Based on Network Path Selection," IEEE JSAC, vol. 24, no. 3, Mar. 2006, pp. 659-72.

[18] Oh-Soon Shin, M. Chan, H.T. Kung, and Vahid Tarokh, "Design of an OFDM Cooperative Space-Time Diversity System," IEEE Trans. On Veh. Technology, Vol. 56, No.4, July 2007.

[19] Hyundong Shin Jae Hong Lee ," On the error probability of binary and M-ary signals in Nakagami-m fading channels", IEEE Trans. on Communications, Vol.52, No.4, April 2004.

[20] A. Erdelyi, Higher Transcendental Functions. New York: McGraw-Hill, 1953, vol. 1.

[21] Gradsheteyn, I.S., and Ryzhik, I.M.: 'Table of integrals, series, and products' Academic press, New York, Sixth Edition 2000.

[22] J. G. Proakis, Digital Communications, 4th ed. New York: McGraw- Hill, 2001

\section{Author' biography}

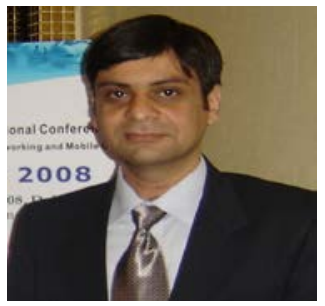

Irfan Ahmed received the B.E. Electrical Engineering degree from University of Engineering and Technology, Taxila, Pakistan, in 1999, the M.S. Computer Engineering degree from CASE, Islamabad, Pakistan, in 2003, and the PhD degree in Telecommunication Engineering from Beijing University of Posts and Telecommunications, Beijing, China, in 2008.

Currently, he is working as assistant professor in Taif University, KSA. He was post-doctoral fellow with Qatar University from April 2010 to March 2011, where he worked on two research projects, wireless mesh networks with Purdue University, USA, and radio resource

allocation for LTE with Qtel. He has also been involved in National ICT Pakistan funded research project "design and development of MIMO and Cooperative MIMO test-bed" at Iqra University, Islamabad, Pakistan, during 2008 to 2010 . His research interests include wireless LAN (WLAN) medium access control (MAC) protocol design and analysis, cooperative communications, MIMO communications, performance analysis of wireless channels, energy constrained wireless networks, cognitive radio networks, and radio resource allocation. He is an author of more than 15 International publications.

Dr. Irfan served as technical program committee member of FIT 2012 conference and session chair of the IEEE Wireless Communications, Networking and Mobile Computing conference. He is an active reviewer of IEEE Transaction, Springer and Elsevier journals, and IEEE ICC, VTC conferences. 